\title{
A CCAAT-binding factor, SINFYA10, negatively regulates ascorbate accumulation by modulating the D-mannose/L-galactose pathway in tomato
}

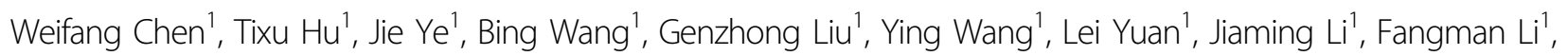
Zhibiao $Y e^{1,2}$ and Yuyang Zhang $\mathbb{B}^{1,2}$

\begin{abstract}
Ascorbic acid (AsA), an important antioxidant and growth regulator, and it is essential for plant development and human health. Specifically, humans have to acquire AsA from dietary sources due to their inability to synthesize it. The AsA biosynthesis pathway in plants has been elucidated, but its regulatory mechanism remains largely unknown. In this report, we biochemically identified a CCAAT-box transcription factor (SINFYA10) that can bind to the promoter of SIGME1, which encodes GDP-Man-3',5'-epimerase, a pivotal enzyme in the D-mannose/L-galactose pathway. Importantly, SINFYA10 simultaneously binds to the promoter of SIGGPI, a downstream gene of S/GME1 in the Dmannose/L-galactose pathway. Binding assays in yeast and functional analyses in plants have confirmed that SINFYA10 exerts a negative effect on the expression of both SIGME1 and SIGGP1. Transgenic tomato lines overexpressing SINFYA10 show decreased levels of SIGME1 and SIGGP1 abundance and AsA concentration in their leaves and fruits, accompanied by enhanced sensitivity to oxidative stress. Overall, SINFYA10 is the first CCAAT-binding factor identified to date to negatively regulate the AsA biosynthetic pathway at multiple sites and modulate plant responses to oxidative stress.
\end{abstract}

\section{Introduction}

Ascorbic acid (AsA, also referred to as vitamin C) contributes to nutritional quality and stress responses in plants and thus has attracted increased amounts of attention. Since humans and higher primates have lost the capability to synthesize AsA, this compound must be obtained from dietary sources rich in AsA, e.g., fresh vegetables and fruits ${ }^{1}$. AsA plays an important role in protecting human health and in disease prevention. For example, AsA can effectively control tumor progression ${ }^{2}$, and intracellular AsA helps maintain the integrity and function of several processes in the central nervous system $^{3}$. In plants, AsA can remove reactive oxygen species produced by photosynthesis, respiration, biological stress,

Correspondence: Yuyang Zhang (yyzhang@mail.hzauedu.cn)

${ }^{1}$ Key Laboratory of Horticultural Plant Biology, Ministry of Education, Huazhong Agricultural University, 430070 Wuhan, China

${ }^{2} \mathrm{HZAU}$ Chuwei Institute of Advanced Seeds, 430070 Wuhan, China and abiotic stress so that plants can carry out normal physiological processes. For AsA biosynthesis, the major biosynthesis pathway is the D-mannose/L-galactose pathway (Wheeler \& Smirnoff pathway), although an alternative pathway in plants involving galacturonate has been proposed $^{4,5}$. However, controversy exists between the Dglucose $^{6}$ and myo-inositol pathways ${ }^{7}$. Between them, the pivotal biosynthetic pathway of D-mannose/L-galactose involves ten catalytic steps through which D-glucose is converted to AsA. The first six steps involved the synthesis of active riboside sugars, which are utilized as substrates for AsA synthesis, as well as precursors of cell wall polysaccharides and glycoproteins. The last four steps, starting from GDP-L-galactose, constitute the exclusive steps for AsA synthesis. The genes in the D-Man/L-Gal pathway have been identified; they encode GDP-D-Man pyrophosphorylase $(\mathrm{GMP})^{8}$, GDP-Man-3',5'-epimerase $(\mathrm{GME})^{9}$, GDP-L-Gal phosphorylase $(\mathrm{GGP})^{10}$, L-Gal-1-P

\section{(c) The Author(s) 2020}

(c) (i) Open Access This article is licensed under a Creative Commons Attribution 4.0 International License, which permits use, sharing, adaptation, distribution and reproduction c. in any medium or format, as long as you give appropriate credit to the original author(s) and the source, provide a link to the Creative Commons license, and indicate if changes were made. The images or other third party material in this article are included in the article's Creative Commons license, unless indicated otherwise in a credit line to the material. If material is not included in the article's Creative Commons license and your intended use is not permitted by statutory regulation or exceeds the permitted use, you will need to obtain permission directly from the copyright holder. To view a copy of this license, visit http://creativecommons.org/licenses/by/4.0/. 
phosphatase $(\mathrm{GPP})^{11}$, L-Gal dehydrogenase $(\mathrm{GalDH})^{12}$, and L-galactono-1,4-lactone dehydrogenase $(\mathrm{GLDH})^{13}$. GME converts GDP-D-mannose into GDP-L-galactose, yielding an alternative product of GDP-L-glucose. In most plants, there is a single copy of the $G M E$ gene $^{14}$, whereas in tomato (Solanum lycopersicum), two GME genes named GME1 and GME2 have been found ${ }^{15}$. Overexpression of GME1 and GME2 could increase the AsA content, while knockdown of either GME gene decreased the AsA content ${ }^{16}$. Simultaneous suppression of the GME1 and GME2 genes by RNA interference (RNAi) could reduce the AsA content by $40-60 \%$, with increasing cell wall mannose content and fragility ${ }^{17}$. Suppressed expression of GME1 or GME2 separately revealed different functions in cell wall synthesis ${ }^{18}$, suggesting the divergence of functions of $G M E$ family members.

In plants, the AsA content is affected by various factors, such as light ${ }^{19,20}$, temperature ${ }^{21}$, minerals $^{22}$, and environmental cues. AsA accumulation is also regulated by hormones such as auxin ${ }^{23}$, methyl jasmonate ${ }^{24}$, jasmonic $\operatorname{acid}^{25}$, and Abscisic Acid (ABA) ${ }^{26}$. Transcription factors or regulators purportedly modulate AsA biosynthesis. amr1 (Ascorbic acid Mannose Pathway Regulator 1), an activation-tagged Arabidopsis (Arabidopsis thaliana) ozone-sensitive mutant, showed decreased AsA content when the expression of genes in the D-Man/L-Gal pathway were negatively regulated ${ }^{27}$. The photomorphogenic factor COP9 signalosome subunit 5B (CSN5B) could decrease the AsA content by modulating the protein abundance of the AsA biosynthetic enzyme GDP-Manpyrophosphorylase (VTC1) ${ }^{28}$. Arabidopsis AtERF98 and the tomato HD-Zip I family transcription factor (SlHZ24) could bind to the VTC1 promoter and increased the AsA content by positively regulating VTC1 expression ${ }^{29,30}$. Two nucleotide sugar pyrophosphorylase-like proteins from Arabidopsis, KONJAC1 (KJC1) and $\mathrm{KJC} 2$, could stimulate GMP enzymatic activity and increase AsA accumulation $^{31}$. Moreover, a Cys2/His2-type zinc-finger protein named SIZF3 could directly interact with CSN5B, preventing CSN5B binding to GMP and thus increasing the AsA content in tomato ${ }^{32}$. More recently, an upstream open reading frame (uORF) in the long untranslated region of GGP was demonstrated to encode a peptide that functions in the feedback regulation of AsA at the posttranscriptional level ${ }^{33}$. To date, the regulation of AsA biosynthesis largely occurs at the VTC1-catalyzed step ${ }^{28-30,32}$, while the regulation of other genes in the AsA synthesis pathway deserves further investigation.

NFYAs belong to the NFY (Nuclear Factor Y, also known as Heme activator protein or CCAAT-binding factor) complex, which contains NFYAs, NFYBs and NFYCs, and function as heterodimers. After dimerization between NFYB and NFYC in the cytoplasm, NFYA is further recruited to form a heterotrimer. NFYA binds to the CCAAT-box to regulate the expression of downstream genes ${ }^{34}$. Sequences associated with the NFY heterotrimer complex have been found in all the sequenced genomes of eukaryotes ${ }^{35}$, and there are one or two genes encoding NFYs in mammals and yeast ${ }^{36}$. However, in plants, the NFY family has undergone expansion ${ }^{34}$. The NFY complex was reported to be involved in the regulation of plant growth and development and the stress response. NFYA in wheat positively responds to low nitrogen and phosphorus: overexpression of TaNFYA-B1 stimulated root development by upregulating nitrate and phosphate transporters and, subsequently, nitrogen and phosphorus absorption in roots ${ }^{37}$. Overexpression of the transcription factor NFYC9 mediates ABA signaling by targeting ABA-responsive transcription factors such as $\mathrm{ABI} 5^{38}$. The expression of NFYA5 was strongly induced by drought in an ABA-dependent manner, and NFYA5 overexpression could reduce moisture loss and drought sensitivity ${ }^{39}$. However, overexpression of the wheat NFYA10 gene increased plant sensitivity to salinity, as judged on the basis of seed germination and root growth ${ }^{40}$.

NFY factors have been known to function as heterotrimer complexes during various processes of plant growth and development ${ }^{41-43}$, although only a single NFY transcription factor has been reported to be involved in the stress response in plants. However, the regulatory mechanism through which metabolites, e.g., AsA, accumulate has not been elucidated. In this study, an NFYA transcription factor was determined to negatively regulate AsA biosynthesis at multiple sites in the D-mannose/Lgalactose pathway.

\section{Materials and methods Yeast one-hybrid}

A yeast one-hybrid assay was carried out via a Matchmaker One-Hybrid Library Construction and Screening Kit (Clontech, http://www.clontech.com/) according to the manufacturer's instructions. A 2-kb promoter fragment of SlGME1 from the initiation site, as well as a mutated fragment of the SlGME1 promoter were amplified from tomato genomic DNA and then cloned into a pAbAi vector (Clontech). The full-length and truncated NFYA CDSs were amplified from tomato complementary DNA (cDNA) via PCR and cloned into a pGADT7 vector (Clontech); the primer sequences used are listed in Supplementary Table S2. Both the pHIS2.0 bait vector and the pGADT7 prey vector were introduced into Y1H Gold yeast (Clontech) and cultured on SD/-Leu-Ura media. After 3 days, the positive yeast strains were selected and diluted in sterilized distilled water to an $\mathrm{OD}_{600}$ of 0.1 , and $2.5 \mu \mathrm{l}$ of suspension was spotted onto SD/-Leu-Ura media, with or without $\mathrm{AbA}$ (Clontech). The plates were subsequently incubated for $3-7$ days at $30^{\circ} \mathrm{C}$. 
Transient expression in tobacco leaves by agro infiltration

There are four CCAAT-boxes in the 2-kb promoter of SlGME1, and different 5'-deleted SlGME1 promoter fragments were amplified with specific primers (Supplementary Table S2). These fragments were cloned into a pGreenII 0800-LUC reporter vector. The full-length ORF of NFYA was amplified from tomato cDNA and inserted into a pGreenII 62-SK effector vector, which was subsequently introduced into $A$. tumefaciens strain GV3101 cells together with a pSoup helper vector. The Agrobacterium cells were subsequently activated by treatment with AI buffer $(10 \mathrm{mmol} / \mathrm{l}$ MES, $10 \mathrm{mmol} / \mathrm{l}$ $\mathrm{MgCl}_{2}, 150 \mu \mathrm{mol} / \mathrm{l} \mathrm{AS}$ ). The Agrobacterium cells with different vectors were mixed together and injected into young tobacco (Nicotiana benthamiana) leaves for transient expression, after which they were were evaluated after a 2-day incubation period ${ }^{44}$. Luciferase reporter genes were assayed using a Dual-Luciferase Reporter Assay System (Promega, http://www.promega.com/) using an Infinite M200 Pro instrument (Tecan).

A 35S::UAS-GUS reporter system was used to characterize the transcriptional activity of SINFYA10, as described by Wang et al. ${ }^{45}$. The CDS of SINFYA10 was amplified and linked to a pYF503 vector, generating a GDBD-SINFYA10 effector construct. The effector construct, empty control (pYF503, designated GDBD), and 35S::UAS-GUS reporter plasmid were then transformed into A. tumefaciens strain GV2260. The reporter and two effectors were mixed together, after which the mixture injected into tobacco leaves, followed by GUS staining after a $48 \mathrm{~h}$ incubation period. GUS staining was performed as described previously ${ }^{46}$.

\section{Vector construction and tomato transformation}

Full-length NFYA ORFs and RNAi fragments were amplified via PCR using Phanta polymerase (Vazyme, Nanjing, China) from tomato cDNA using gene-specific primers (Supplementary Table S2). The full-length SINFYA10 ORF and RNAi fragment were then cloned into a pMV3 overexpression vector (modified from pHELLSGATE8) and pHELLSGATE8 (Invitrogen), respectively, via recombination using Exnase II. These vectors were transferred into A. tumefaciens strain C58 for tomato transformation. The constructs were subsequently transformed into Ailsa Craig (AC) tomato, and transgenic plants were confirmed via PCR using genomic DNA from the leaves; the primers used are shown in Supplementary Table S2.

\section{Gene expression analysis}

Total RNA was extracted from roots, stems, leaves, and flowers as well as immature fruit, green fruit, breakerstage fruit, and red ripe fruit using TRIzol reagent. The RNA was then reverse transcribed into cDNA using a
HiScript II 1st Strand cDNA Synthesis Kit (Vazyme), and the gene expression in these tissues was measured. Quantitative PCR (qPCR) was performed using a SYBR Light Cycler 480 instrument in conjunction with SYBR Green I Master Kit (Roche, http://www.roche.com/) according to the manufacturer's protocols. The RNA of the transgenic plants and wild type was extracted and reverse transcribed to cDNA to determine the expression of SINFYA10, SlGME1, SlGME2, and other genes in the AsA biosynthesis pathway. The sequences of the primers used are listed in Supplementary Table S3. Three biological replicates for the transgenic lines and the wild type were evaluated. The relative expression of specific genes was quantified using the $2^{-\Delta \Delta \mathrm{Ct}}$ method, and the actin gene was used as a constitutive internal control.

\section{Ascorbic acid assays}

The AsA levels were measured in $\mathrm{T}_{1}$ transgenic lines, as well as wild-type plants as previously described ${ }^{47}$. Leaf samples were taken from the third or fourth leaf from the top of 1-month-old tomato plants. The fruit samples were taken at the red ripe stage. The samples were collected and ground to a fine powder in liquid nitrogen. Approximately $0.2 \mathrm{~g}$ of frozen leaf tissue and $0.3 \mathrm{~g}$ of frozen fruit tissue were added to one milliliter of ice-cold 6\% trichloroacetic acid in a 2-ml Eppendorf tube. Each homogenate was centrifuged at $16,000 \times g$ for $10 \mathrm{~min}$ at $4^{\circ} \mathrm{C}$, after which the supernatant was transferred to a new clean Eppendorf tube. To assay total AsA levels, $20 \mu \mathrm{l}$ of the supernatant was transferred to the wells of a microtiter plate containing $20 \mu \mathrm{l}$ of $5 \mathrm{mmol}$ dithiothreitol (DTT). The plate was incubated for $20 \mathrm{~min}$ at $37^{\circ} \mathrm{C}$ to convert the oxidized ascorbic acid into the reduced form. Ten microliters of N-ethylmaleimide (NEM; $0.5 \% \mathrm{w} / \mathrm{v}$ in water) was added to remove the excess DTT, followed by incubation for $1 \mathrm{~min}$ at room temperature (approximately $25^{\circ} \mathrm{C}$ ). Eighty microliters of the color reagent (see below) was then added to the mixture, followed by incubation for $1 \mathrm{~h}$ at $37^{\circ} \mathrm{C}$. The absorbance was subsequently recorded at $550 \mathrm{~nm}$ using an Infinite M200 Pro instrument (Tecan; http://www.tecan.com/). The color reagents were prepared as follows: solution A consisted of $31 \%$ orthophosphoric acid, $4.6 \%(\mathrm{w} / \mathrm{v})$ TCA and $0.6 \%(\mathrm{w} / \mathrm{v})$ iron chloride $\left(\mathrm{FeCl}_{3}\right)$; solution B consisted of $4 \%$ 2,2-dipyridyl ( $\mathrm{w} / \mathrm{v}$ in $70 \%$ ethanol); and solutions A and B were mixed at a ratio of 2.75:1 before use. To assay reduced AsA, the DTT and NEM were replaced with the same volume of $0.4 \mathrm{M}$ potassium phosphate buffer ( $\mathrm{pH}$ 7.4), while the rest of the procedure was the same as that for the total AsA assay.

\section{Subcellular localization and tissue-specific expression}

The coding sequence without the terminal codon of SINFYA10 was amplified and inserted into the 5' terminus of GFP in a pCAMBIA-1302 vector under the control of 
the CaMV35S promoter to generate 35S::SINFYA10-GFP constructs. Plasmids were extracted and transferred into tobacco protoplasts, and subcellular localization was observed as described previously ${ }^{48}$.

The promoters of SLGME1 and SINFYA10 were amplified and cloned into a pMV2 vector harboring the GUS reporter gene, and the resultant vectors were transferred into $A$. tumefaciens C58 for tomato transformation. GUS staining was performed on seedlings, flowers and mature green fruits.

\section{Oxidative stress experiments}

To evaluate the oxidative resistance of SINFYA10overexpressing and knockdown lines and the wild type, $80 \mu \mathrm{l}$ of methylviologen (MV, dissolved in water consisting of $0.1 \%$ Tween-20) or water consisting of $0.1 \%$ Tween20 (control) was sprayed onto one-month-old seedlings once a day for three consecutive days. The plant phenotypes were observed one week after spraying, and images were collected.

\section{DAB staining}

MV treatments induce cells to produce excessive ROS, resulting in oxidative damage to plants. Leaves from tomato $\mathrm{T}_{2}$ transgenic lines and wild-type plants were treated with MV (or water as a control) to investigate the tomato response to oxidative stress. The MV- and watertreated tomato leaves were soaked in $1 \mathrm{mg} / \mathrm{ml} \mathrm{DAB}$ solution $(\mathrm{pH}=3.8)$ and then incubated in darkness at room temperature for $24 \mathrm{~h}$. The leaves were then transferred to $96 \%$ ethanol and placed in a boiling water bath for $10 \mathrm{~min}$ to remove the chlorophyll. The ethanol solution was discarded, and the leaves were soaked with $96 \%$ ethanol to remove the floating color (the decolorized leaves were stored in $96 \%$ ethanol). The reactive oxygen species of the decolorized leaves were evaluated visually according to the brownness of the leaves.

\section{Quantification of MDA concentration}

Malondialdehyde (MDA), the final product of lipid peroxidation of the cell membrane, reflects the plant response to oxidative stress. Leaves and ripe fruits from $\mathrm{T}_{2}$ transgenic lines and wild-type plants were utilized to measure the MDA concentration. Samples were collected and ground to a fine powder in liquid nitrogen, followed by the addition of $3 \mathrm{ml}$ of $5 \%$ TCA to $0.2 \mathrm{~g}$ of ground tissue; the extraction was performed at room temperature for $30 \mathrm{~min}$. After centrifugation, $2 \mathrm{ml}$ of the supernatant was transferred into a $10 \mathrm{ml}$ tube, after which $2 \mathrm{ml}$ of $0.67 \%$ TBA solution was added. After blending, the absorption value of the $200 \mu \mathrm{l}$ mixture was measured at wavelengths of $450 \mathrm{~nm}, 532 \mathrm{~nm}$ and $600 \mathrm{~nm}$. The supernatant in the standard solution was replaced with $\mathrm{ddH}_{2} \mathrm{O}$. The concentration of MDA was calculated using the formula $C=6.45 \times\left(\mathrm{OD}_{532}-\mathrm{OD}_{600}\right)-0.56 \times \mathrm{OD}_{450}$.

\section{Determination of POD activity}

Using $\mathrm{H}_{2} \mathrm{O}_{2}$ as an oxidant, POD (peroxidase) catalyzes redox reactions, scavenging $\mathrm{H}_{2} \mathrm{O}_{2}$ from cells. POD activity can reflect the antioxidant capacity and stress tolerance of plants. Leaves and ripe fruits from $\mathrm{T}_{2}$ transgenic lines and wild-type plants were harvested to measure POD activity. Samples were collected and ground to a fine powder in liquid nitrogen, followed by the addition of $800 \mu \mathrm{l}$ of precooled phosphate buffer ( $50 \mathrm{mmol} / \mathrm{l}, \mathrm{pH} 7.8)$, mixing and centrifugation. The supernatant $(20 \mu \mathrm{l})$ was subsequently added to a $1.5 \mathrm{ml}$ reaction mixture, which consisted of $50 \mathrm{ml}$ of phosphate buffer $(200 \mathrm{mmol} / \mathrm{l}, \mathrm{pH} 6.0)$, $28 \mu \mathrm{l}$ of 2 -methoxyphenol and $19 \mu \mathrm{l}$ of hydrogen peroxide $(30 \%)$. The absorption value of the mixture was measured at a wavelength of $470 \mathrm{~nm}$.

\section{Results}

A CCAAT-box transcription factor, SINFY10, is a candidate regulator of ascorbic acid biosynthesis

GME1 and GME2 are two homologous genes in tomato. Both contain six exons and five introns, and the first and fourth introns of GME2 are longer than those of GME1. GME1-overexpressing lines showed higher AsA levels than did GME2-overexpressing lines ${ }^{16}$. Therefore, we used the promoter of GME1 to perform a yeast onehybrid assay in an equalized tomato cDNA library comprising various tissues of roots, leaves, flowers, and fruits at different stages to identify the candidate proteins binding to the promoter of GME1. The potential transcription factors or binding proteins involved in GME1 promoter binding and AsA regulation were isolated (Supplementary Table S1).

Among the candidate binding proteins, the only annotated transcription factor, a CCAAT-box transcription factor (Solyc01g006930.2) that belong to the NFYA family and is a subunit of the NF-Y complex, was further investigated. The sequence of Solyc01g006930.2 was queried via BLAST against the SOL Genomics Network database (http://solgenomics.net/) and mapped onto chromosome 1. It contains an open reading frame of $936 \mathrm{bp}$, encoding $312 \mathrm{aa}$, with an NFYB/NFYC interaction domain (A1: 145-163 aa) and a CCAAT-binding domain (A2: 175-198 aa) (Fig. 1a), and its sequence is highly homologous with that of its counterparts from other plant species (Fig. 1b). According to phylogenetic evolution and annotation, the isolated CCAAT-box transcription factor is hereafter referred to as SINFYA10.

\section{SINFYA10 binds to the SIGME1 promoter in plants and yeast}

To test the hypothesis that SINFYA10 could directly bind to the CCAAT-box in the promoter of GME1, we analyzed the 2-kb promoter fragment of GME1 through the PLACE (http://www.dna.affrc.go.jp/PLACE/ 


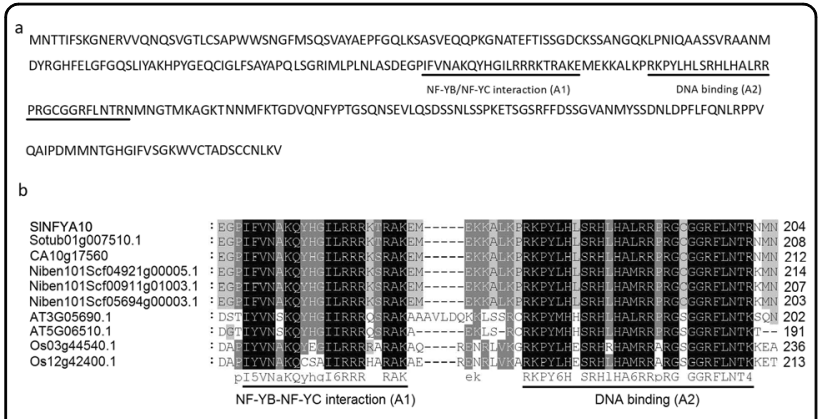

Fig. 1 Alignment of the SINFYA10 sequence with the sequences of its counterparts in plants. a Amino acid sequence of SINFYA10. The NFYB-NFYC interaction (A1) domain and DNA-binding (A2) domain are underlined in black and gray, respectively. $\mathbf{b}$ Amino acid alignment of the conserved domains of SINFYA10 and its counterparts from different species. The protein sequences are from tomato (SINFYA10), potato (Solanum tuberosum: Sotub01g007510.1), pepper (Capsicum annuum: CA10g17560), tobacco (Nicotiana attenuata: Niben101Scf04921g00005.1, Niben101Scf00911 g01003.1, and Niben101Scf05694g00003.1), Arabidopsis (AT5G06510.1 and AT3G05690.1), and rice (Oryza sativa L.: OS_03g44540.1 and OS_12g42400.1)

signalscan.html) and PlantCARE (http://bioinfor-matics. psb.ugent.be/webtools/plantcare/html/) databases ${ }^{49}$. We found four potential target elements, two elements of CCAAT and two of ATTGG, that were named C1 and C2 as well as $\mathrm{C} 3$ and $\mathrm{C} 4$, respectively (Fig. 2a).

NFYA proteins have been previously reported to recognize and bind to CCAAT-box cis-elements, thus regulating the expression of downstream genes. To verify whether SINFYA10 recognizes and binds to one of these cis-elements, we generated five constructs containing serial truncated promoters (Fig. 2a). These constructs were then transiently expressed in tobacco (Nicotiana benthamiana) leaves by A. tumefaciens (GV2260)-mediated transformation. Plant agro infiltration showed that the truncation of the promoter successively reduced the promoter capability and luciferase (LUC) activity. When coinfiltrated together with SINFYA10, the SlGME1 promoter with four boxes (proGME1-1: C1-C4) and three boxes (proGME1-2: C2-C4) showed significantly reduced LUC activity compared to that of the control. However, the promoter fragments with two boxes (proGME1-3: C3-C4), one box (proGME1-4: C4) or no box (proGME1-5) did not show significant differences in LUC activity compared to that of the control (Fig. 2b). These results indicated that SINFYA10 mainly binds to $\mathrm{C} 1$ and $\mathrm{C} 2$ boxes or only $\mathrm{C} 2$ boxes.

To identify the core sequence of the SlGME1 promoter bound by SINFYA10, constructs with truncated promoters containing $\mathrm{C} 1$ and $\mathrm{C} 2$ boxes were used in a yeast one-hybrid assay (Fig. 3a). In addition, we artificially mutated the two boxes and transferred them into yeast Y1H Gold strains. The results showed that the SlGME1

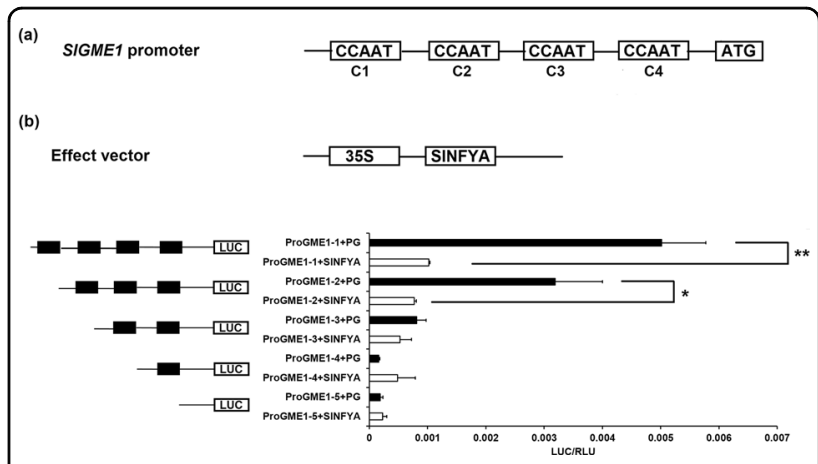

Fig. 2 Interaction of SINFYA10 with the SIGME1 promoter according to a dual-luciferase assay. a CCAAT-box distribution within the SIGMEI 2-kb promoter. There are four cis-elements in the 2 -kb promoter region of SIGME1. Four CCAAT elements are represented by black boxes and are named $C_{1}, C 2, C 3$, and $C 4$. b Dual-luciferase assay between the SIGME1 promoter and SINFYA10. The full sequence and serial truncated versions of the SIGME1 promoter were ligated into pGreenll 0800-LUC as a reporter vector, and SINFYA10 was ligated into pGreenll 62-SK as an effector vector. $P G$, the pGreenll 62-SK empty vector with serial SIGME1 truncated promoters, was used as a control. LUC luciferase activity. RLC Renilla luciferase. The data are means \pm SEs $(N=3)$

promoter containing native $\mathrm{C} 1$ and $\mathrm{C} 2$ boxes could be bound by SINFYA10 and that fragments with mutant $\mathrm{C} 1$ boxes and native $\mathrm{C} 2$ boxes could be bound by SINFYA10. However, the promoter fragments with native $\mathrm{C} 1$ and mutant $\mathrm{C} 2$ boxes or both mutant $\mathrm{C} 1$ and $\mathrm{C} 2$ boxes could not be bound by SINFYA10. Taken together, these results indicated that SINFYA10 may preferably bind to the C2 box of the SIGME1 promoter.

To further investigate the interaction characteristics between SINFYA10 and the core elements of the SlGME1 promoter, we analyzed the protein structure of SINFYA10 via the NCBI database (https://www.ncbi.nlm.nih.gov/) and found that there are two domains: a DNA-binding domain and an NFYB/NFYC interaction domain. According to the protein structure of SINFYA10, we divided the coding sequence into four segments (N1-N4) and inserted them into pGADT7 vectors. We used a yeast one-hybrid assay to test the interaction of these different SINFYA10 segments with the C2 box of the SlGME1 promoter. The results demonstrated that N2, N3, and N4 could recognize and bind to the $\mathrm{C} 2$ box of the SlGME1 promoter, while the $\mathrm{N} 1$ fragment could not (Fig. 3b), indicating that the C-terminus of NFYA is crucial for binding to the $S l G M E 1$ promoter.

We subsequently inserted the promoter of SIGME1 into pMV2 to generate proSlGME1::GUS and cloned SINFYA10 into pHELLSGATE8 to generate a pro35S:: SINFYA10 overexpression construct. We transferred the two vectors into A. tumefaciens (GV2260) and mixed them together for agro infiltration of tobacco leaves, after which GUS staining and GUS gene expression 


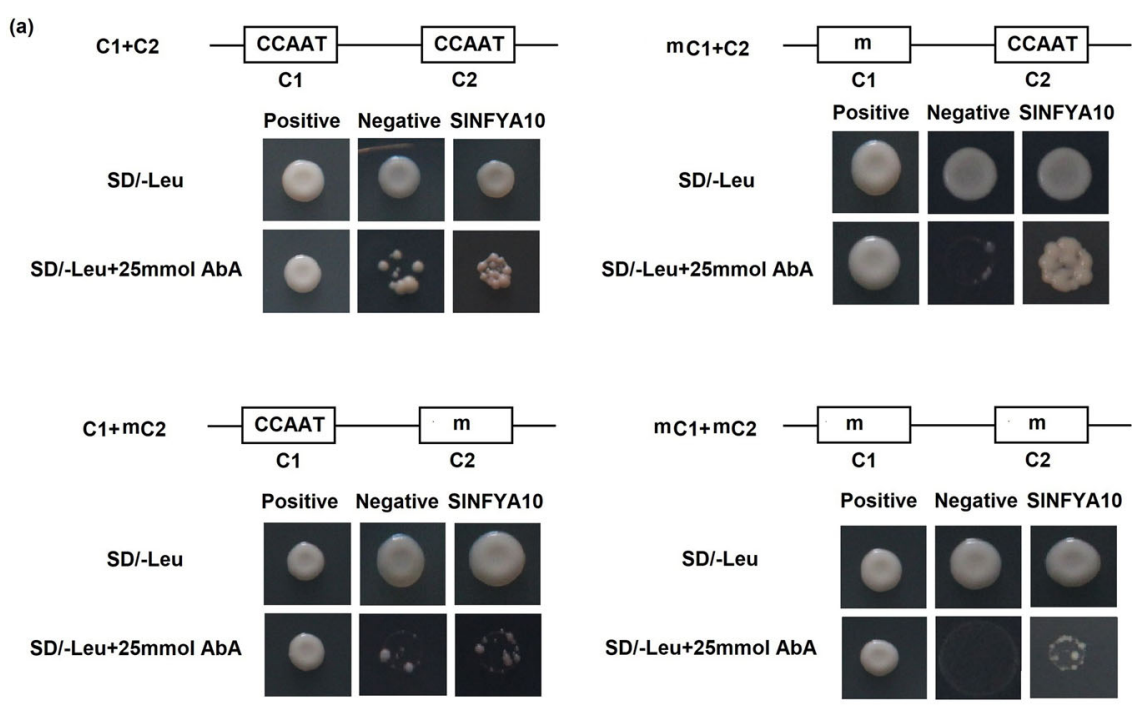

(b)

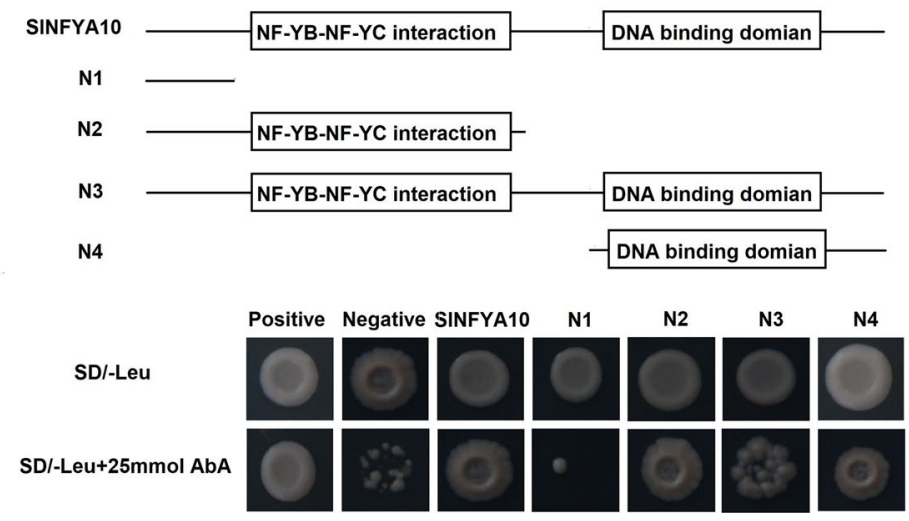

Fig. 3 SINFYA10 binds to the SIGME1 promoter. a The SIGME1 promoter fragment harboring C1 and C2 elements interacts with SINFYA10 according to a yeast one-hybrid assay. The $\mathrm{C} 1$ and $\mathrm{C} 2$ elements along with their mutated version constituted four combinations (the first contained native $\mathrm{C} 1$ and $\mathrm{C} 2$ elements, the second contained mutated $\mathrm{C} 1$ and native $\mathrm{C} 2$ elements, the third contained native C1 elements and mutated C2 elements, and the fourth contained mutated $\mathrm{C} 1$ and $\mathrm{C} 2$ elements). These promoter segments were inserted into the pAbAi vector as bait, and SINFYA10 was inserted into the pGADT7 vector as prey. The bait and prey vectors were cotransformed together along with a negative control (baitpGADT7) or positive control (p53-AbAi/pGAD-p53) into yeast on selective media with or without antibiotics ( $25 \mathrm{ng} / \mathrm{ml}$ AbA). a The interaction between C2 elements and serial SINFYA10 domains according to a yeast one-hybrid assay. On the basis of its structural characteristics, SINFYA10 was divided into four segments and inserted into the pGADT7 vector to serve as prey, and the SIGME1 promoter contained the whole C2 element as bait. The transformed yeast cells were grown on selective media with or without antibiotics $(25 \mathrm{ng} / \mathrm{ml} \mathrm{AbA})$

were evaluated. The tobacco leaves infiltrated with proSlGME1::GUS and pHELLSGATE8 exhibited blue GUS activity, while replacement of pHELLSGATE8 with pro35S::SINFYA10 led to significantly reduced GUS expression. This was confirmed by qPCR-based detection of GUS gene expression in the infiltrated leaves. Taken together, these results indicated that SINFYA10 can interact with and negatively regulated the SlGME1 promoter (Fig. 4).

A GAL4/UAS-based assay was also performed, where GDBD binds to six copies of UAS to activate GUS expression $^{50,45}$. The SINFYA10 coding sequence was fused to GDBD to generate a GDBD-SINFYA10 fusion protein, which was then coexpressed together with 35S-UAS-GUS in tobacco leaves. Histochemical staining showed that GUS expression was prominently repressed in the leaves cotransformed with GDBD-SINFYA10 compared with the leaves cotransformed with 35SGDBD (Supplementary Fig. S1), suggesting that SINFYA10 might act as a transcriptional repressor.

\section{SINFYA10 regulates AsA biosynthesis by modulating SIGME1 expression}

We constructed SINFYA10-overexpressing (CO) and RNAi knockdown (CR) tomato lines. The overexpressing lines exhibited significantly higher SINFYA10 expression 

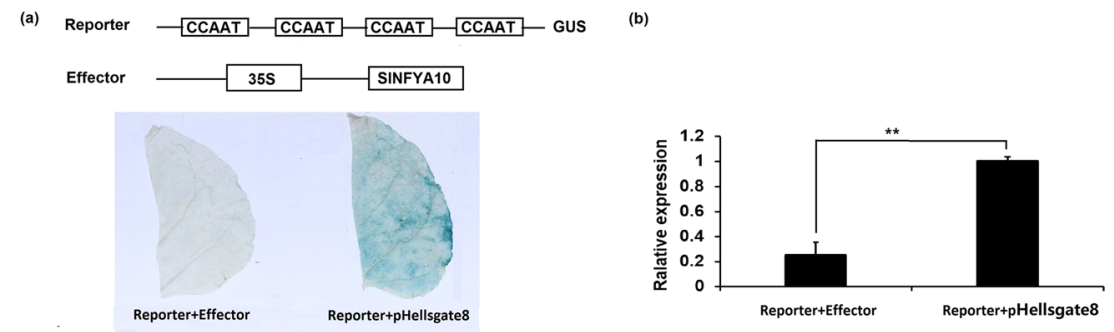

Fig. 4 Transcriptional activity of SINFYA10 in plants. Schematic diagram of the two vectors used for the transient expression assays. The GUS gene driven by the SIGME1 promoter (proSIGME1::GUS), along with the SINFYA10-overexpressing vector as the effector (35S::SINFYA10), were coinfiltrated into tobacco leaves. A GUS reporter plus pHELLSGATE8 empty vector was utilized as a control. GUS staining (a) and GUS gene expression (b) were evaluated. The asterisks indicates significant differences ${ }^{* *} P \leq 0.01$ )
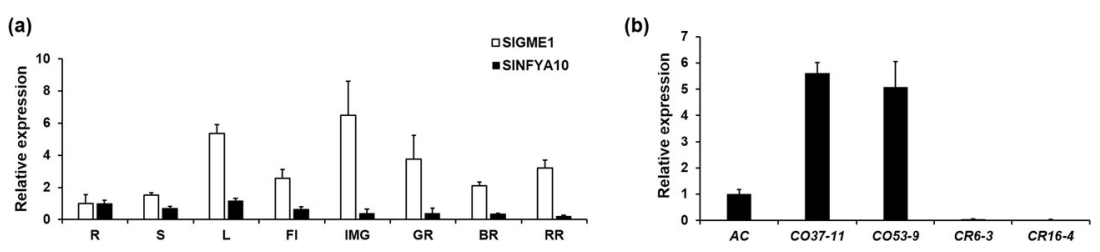

Fig. 5 Relative expression of SIGME1 and SINFYA10 in tomato. a Tissue expression of SIGME1 and SINFYA10. SIGME1 and SINFYA10 expression in different organs: R roots, S stems, L leaves, FI flowers, IMG immature fruits, MG mature green fruits, BR breaker-stage fruits, RR red ripe fruits. b Relative expression level of SINFYA10 in transgenic lines. QPCR-based analysis was performed on two SINFYA10-overexpressing lines (CO37-11, CO53-9) and two RNAi lines (CR6-3, CR16-4). The data are presented as the means \pm SEs

than did the wild-type control, while the CR lines showed a decrease in expression. The expression of SINFYA10 in the leaves of the CO-37-11 and CO-53-9 lines was 5.6and 5.0-fold that in wild-type leaves, while the CR-6-3 and CR-16-4 lines showed decreased expression (4\% and $16 \%$ lower than that of the wild-type control, respectively) (Fig. 5b). The AsA levels of the transgenic lines and the wild-type plants were assayed, and the results revealed a significant decrease in the leaves and red ripe fruits of the overexpression lines (Fig. 6a, b), while the RNAi transgenic lines showed no difference in AsA levels (Supplementary Fig. S2a, b). This might be due to the functional redundancy of the NFYA gene family members, for which suppression of a single gene member might be compensated by alternative gene members.

It was previously reported that overexpression of the AsA biosynthetic gene SlGME1 in tomato caused an increase in AsA levels ${ }^{16}$. We found that enhanced expression of SINFYA1O modulated its target gene, SlGME1, in SINFYA10-overexpressing plants. The expression of SlGME1 was significantly decreased in the leaves and red ripe fruits of the overexpression lines compared with the wild type (Fig. 6c, d). However, SlGME1 expression was not altered in the RNAi lines (Supplementary Fig. S2c, d). In the SINFYA10 transgenic lines, SlGME2 expression was unchanged (Fig. 6c, d and Supplementary Fig. S2c, d). Yeast one-hybrid assays between the SlGME2 promoter and SINFYA10 consistently showed no interaction (Supplementary Fig. S3). The negative regulatory effects of SINFYA10 on SlGME1 expression were also supported by the fact that SINFYA10 and SlGME1 expression abundance exhibited opposite change patterns among the different tissues (Fig. 5a). In summary, SINFYA10 exerts a specific regulatory effect on SlGME1 and subsequently negatively modulates AsA accumulation.

\section{SINFYA10 regulates multiple sites in the D-mannose/L- galactose pathway}

To identify whether SINFYA10 regulates the expression of other biosynthetic genes in the D-Man/L-Gal pathway, we performed $\mathrm{qPCR}$ on the leaves and red ripe fruits of the SINFYA10 transgenic lines. In the leaves and red ripe fruits, the expression of SlGGP1 was decreased in the CO lines and enhanced in the CR lines (Supplementary Fig. S4). The pattern of SlGGP1 expression modulated by SINFYA10 in the transgenic lines was similar to that of SIGME1 expression. These results indicate that SINFYA10 might regulate AsA biosynthesis at multiple sites.

We retrieved the sequences of the promoter fragments of AsA biosynthetic genes from the SOL Genomics Network database and analyzed their cis-elements using the PLACE program. Some genes (e.g., SlGGP, SlGME2, $S l G L D H)$ were predicted to contain CCAAT-boxes that might be bound with NFYA proteins (Supplementary Table S4). We tested the potential interaction between 

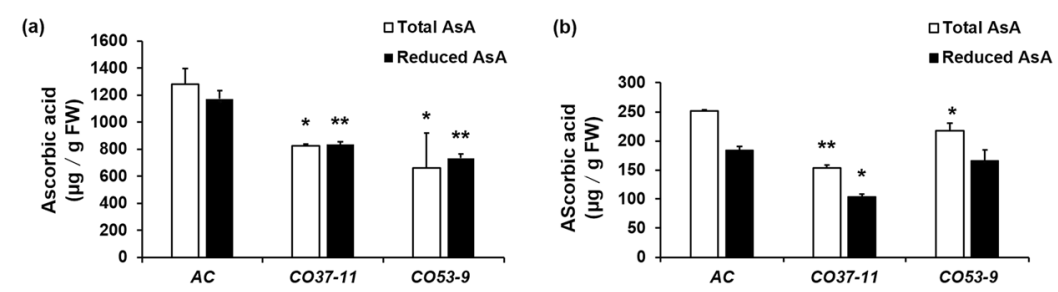

(c)

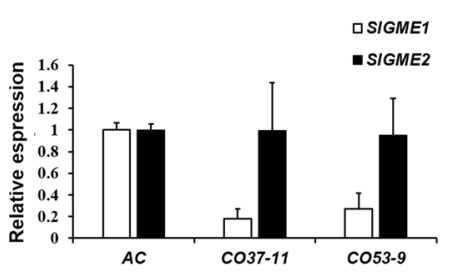

(d)

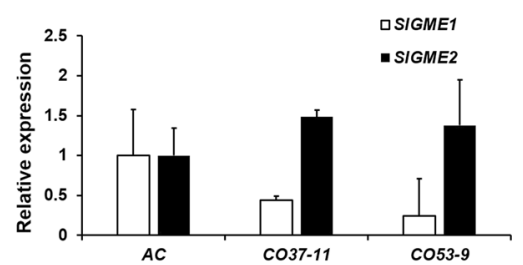

Fig. 6 Ascorbic acid levels and SIGME expression in SINFYA10 transgenic tomato lines. a Total and reduced ascorbic acid content in young leaves of SINFYA10-overexpressing lines. b Total and reduced ascorbic acid content in red ripe fruits of SINFYA10-overexpressing lines. c Relative expression levels of SIGME1 and SIGME2 in young leaves of SINFYA10-overexpressing lines. d Relative expression levels of SIGME1 and SIGME2 in red ripe fruits of SINFYA10-overexpressing lines. The data are the means \pm SEs $(N=3)$, and the asterisks indicate significant differences (** $P \leq 0.01$; $* P \leq 0.05)$

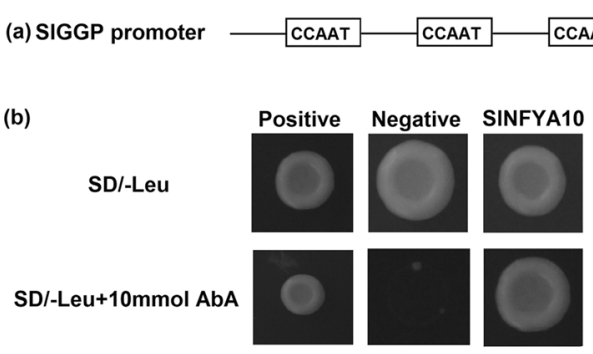

\section{CCAAT CCAAT CCAAT ATG}

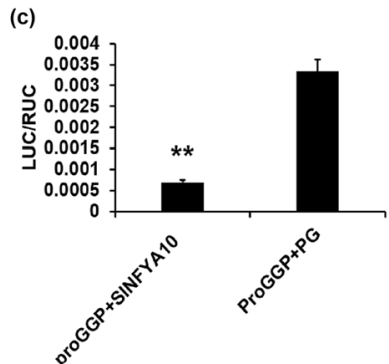

Fig. 7 SINFYA10 binding to the SIGGP1 promoter. a Analysis of CCAAT elements in the $1.5 \mathrm{~kb}$ SIGGP1 promoter region. $\mathbf{b}$ Interaction between the SIGGP1 promoter and SINFYA10 in a yeast one-hybrid assay. The SIGGP1 promoter was inserted into a pAbAi vector as bait, and SINFYA10 was inserted into pGADT7 as prey. Cotransformed yeast cells were grown on selective media with or without $10 \mathrm{ng} / \mathrm{ml} \mathrm{AbA}$. c Transient expression in tobacco leaves. The SIGGP1 promoter was ligated into pGreenll 0800-LUC as a reporter vector, and SINFYA10 was ligated into pGreenII 62-SK as an effector vector. PG, pGreenll 62-SK empty vector

these gene promoters and SINFYA10 by yeast one-hybrid assays. The yeast one-hybrid results showed that SINFYA10 could bind to the promoter of SlGGP1 in yeast (Fig. 7a, b). The SlGGP1 promoter was then inserted into a pGreenII 0800-LUC vector for transient expression in tobacco. Compared with those infiltrated with proSlGGP1 and pGreenII 62-SK, tobacco leaves coinfiltrated with proSlGGP1 and SINFYA10 showed significantly decreased LUC activity (Fig. 7c), suggesting negative regulatory effects of SINFYA10 on SlGGP1 as well as SlGME1.

\section{Nuclear factor SINFYA10 showed tissue-specific expression coincident with SIGME1}

To observe the expression patterns of SlGME1 and SINFYA10, 2-kb length promoters of SlGME1 and
SINFYA10 were inserted into GUS expression vectors separately. The two GUS constructs were subsequently transformed into the tomato AC cultivar. GUS staining was performed on the roots, stems, leaves, flowers, and breaker fruits of proSlGME1::GUS and proSINFYA10:: GUS transgenic lines. From these staining results, we found that both SlGME1 and SINFYA10 are expressed in the roots, stems, flowers and seeds (Fig. 8b).

To investigate the subcellular localization of SINFYA10, a SINFYA10::GFP fusion protein was constructed and transiently expressed in tobacco protoplasts, followed by observations via confocal microscopy. The results showed that SINFYA10 was localized in the nucleus (Fig. 8a), which is consistent with the fact that it is a nuclear transcription factor. 


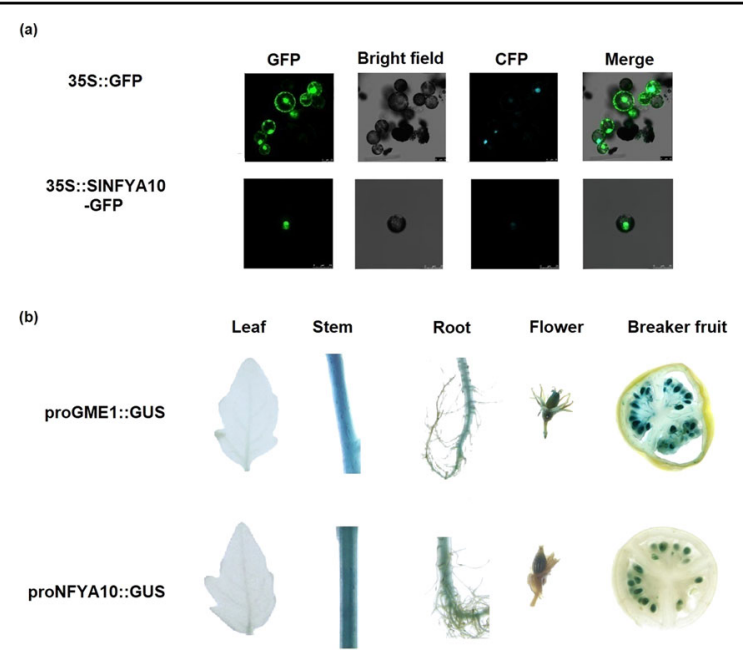

Fig. 8 Nuclear localization of SINFYA10 and its co-occurrence with SIGME1. a Subcellular localization of SINFYA10 in tobacco protoplasts. A GFP tag was fused to SINFYA10, and the fusion protein was transiently expressed in tobacco protoplasts followed by fluorescence observations via confocal microscopy. CFP nuclear marker fluorescence. $\mathbf{b}$ GUS staining of transgenic lines harboring proSIGME1::GUS and proSINFYA10:GUS. The promoters of SIGME1 and SINFYA10 were inserted into the pMV2 vector driving the GUS gene, and GUS staining was performed on the roots, stems, leaves, flowers and breaker-stage fruits

\section{SINFYA10 modulates reactive oxygen species accumulation} and the stress response

AsA is an antioxidant that scavenges reactive oxygen species and protects plants from oxidative stress. We measured the MDA concentration and POD activity in the leaves and fruits of transgenic and wild-type plants. The results showed that the MDA concentration in the overexpressing lines were significantly higher than those in the wild type (Fig. 9a, b), while there was not much difference between the RNAi lines and the control (Supplementary Fig. S5a, b). POD activity was also evaluated in the leaves and fruits, and the results showed that the POD activity was significantly lower in overexpression lines than in the wild-type and RNAi lines (Fig. 9c, d and Supplementary Fig. S5c, d).

To test whether alteration of SINFYA10 expression exerts an effect on the plant stress response, transgenic lines and wild-type plants were treated with MV to induce oxidative stress. DAB staining was subsequently carried out to detect ROS in the leaves. We found that the color of the leaves of the SINFYA10-overexpressing lines was darker than that of the RNAi lines and the control, indicating that the leaves of the SINFYA10-overexpressing lines were more severely affected by oxidative stress (Supplementary Fig. S6). Thus, the AsA levels and ROS scavenging capability decreased in SINFYA10-overexpressing lines. It should be noted that suppression of
SINFYA10 did not significantly alter the ROS accumulation (Supplementary Fig. S6), MDA concentration or POD activity (Supplementary Fig. S5), suggesting functional redundancy among members of the NFYA gene family. However, the antioxidant activity of RNAi lines under stress conditions deserves further investigation.

\section{Discussion}

Transcription factors or regulators of AsA biosynthesis have been reported, most of which focus on the regulation of SlGMP. Limiting SlGME1 can exert a large effect on AsA accumulation. Moreover, overexpression and knockdown of SlGME1 can affect AsA content ${ }^{17}$. In the present study, we used the promoter of SlGME1 to identify a CCAAT-binding transcription factor, SINFYA10, which could collectively modulate SlGME1 and SlGGP1 expression and alter the AsA content in tomato.

The CCAAT-binding transcription factor also named Nuclear Factor Y (NFY) and Heme activator protein contains three subunits: NFYA, NFYB and NFYC ${ }^{34,51}$. NFYAs bind to the CCAAT-box in promoters and, in conjunction with NFYB and NFYC subunits, form the NFY transcription complex to modulate the expression of downstream genes. SINFYA10 belongs to the NFY family, whose members have conserved NFYB/NFYC interaction domains and CCAAT-binding domains. There are also conserved NFYB/NFYC interaction domains and DNAbinding domains in SINFYA10 (Fig. 1a). NFYs have been demonstrated to regulate plant growth and development and stress responses: NFYA5 overexpression alleviates moisture loss and drought sensitivity ${ }^{39}$; overexpression of the wheat NFYA10 gene increases plant sensitivity to salinity, as judged from seed germination and root growth ${ }^{40}$; and NFYs can positively regulate flowering by interacting with the FT (FLOWERING LOCUS) promoter ${ }^{41}$.

When the SlGME1 promoter was used as bait for yeast one-hybrid assays, a CCAAT-box transcription factor (SINFYA10) was isolated. Among the four CCAAT-boxes predicted in the SIGME1 promoter, only the second CCAAT-box could interact with SINFYA10, as revealed by luciferase and yeast one-hybrid assays (Figs. 2 and 3). GUS staining and expression quantification through transient expression in tobacco (Fig. 4) and the expression patterns of SINFYA10 and SIGME1 (Fig. 5) indicated that there may be a negative regulation effect between SINFYA10 and SIGME1. Compared with the control plants, the SINFYA10-overexpressing lines presented a lower AsA content in the leaves and fruits (Fig. 6a, b). These results indicated that SINFYA10 exerts a repressive effect on SlGME1 transcription. The expression of alternative enzymatic genes of the AsA biosynthesis pathway was also evaluated in SINFYA10 transgenic lines, among which $S l G G P 1$ expression pattern was altered in a manner similar to how the pattern of SlGME1 was altered 


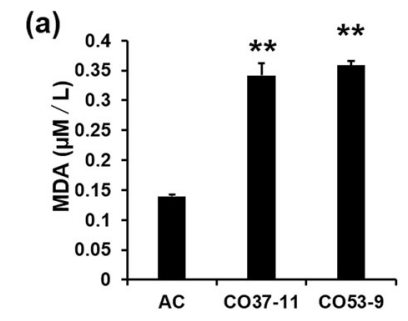

(c)

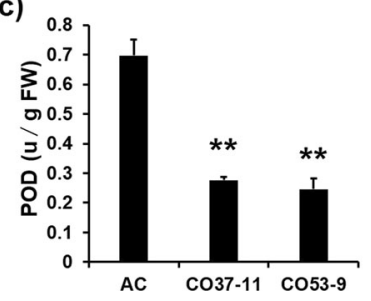

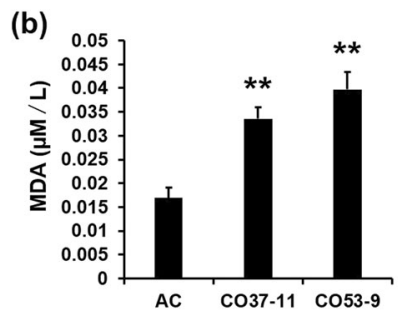

(d)

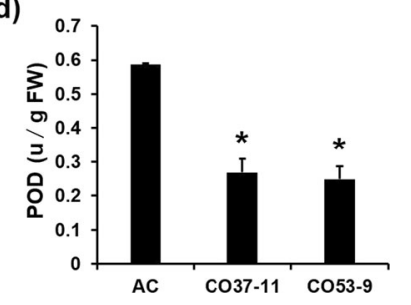

Fig. 9 Antioxidant capacity of SINFYA10 transgenic lines. The MDA concentration was determined in the leaves (a) and ripe fruits (b) of SINFYA10-overexpressing lines and the wild-type control (AC). POD activity was determined in the leaves (a) and red ripe fruits (b) of SINFYA10overexpressing lines and the wild-type control. The data are the means \pm SEs $(N=3)$, and the asterisks indicate significant differences ${ }^{* *} P \leq 0.01$; * $P \leq 0.05)$

(Supplementary Fig. S4). The LUC assay and yeast onehybrid assay showed that SINFYA10 could bind to the SlGGP1 promoter (Fig. 7). In addition, the subcellular localization results showed that SINFYA10 is located in the nucleus, which is characteristic of nuclear transcription factors (Fig. 8a). The tissue distribution of SINFYA10 and SIGME1 showed that they are mainly expressed in the same stem, flower and seed tissues (Fig. 8b). Oxidative stress tests showed that, compared with the control, the SINFYA10-overexpressing lines presented less ROS scavenging activity (Fig. 9 and Supplementary Fig. S6), presumably due to lower availability of AsA, which is the substrate required for ascorbate peroxidase function. Taken together, our results demonstrate that NFYA negatively regulates AsA biosynthesis by suppressing GME1 and GGP1 in tomato fruit. AsA biosynthesis has been widely investigated, and several transcription factors for biosynthetic genes such as GMP have been isolated ${ }^{30}$. In the present work, we confirmed that NFYA could simultaneously regulate GME and GGP expression. NFY transcription factors have been reported to be involved in a wide range of biological processes ${ }^{39-41}$; however, this is the first evidence that NFYA functions in the regulation of AsA metabolism.

It is worth noting that the silencing of SINFYA10 by RNAi did not cause significant alterations in terms of AsA content, SlGME1 and SlGGP1 expression, ROS accumulation, MDA concentration or POD activity in the transgenic lines (Supplementary Figs. S2, S4, S5, S6). Furthermore, fruit development or ripening was not significantly altered in SINFYA10 RNAi transgenic lines compared to the wild type (Supplementary Fig. S7). The unaltered phenotypes of the SINFYA10 RNAi transgenic lines might be due to the functional redundancy of the members of the NFYA gene family. Unlike the NFYAs encoded by one or two genes in animals and yeast, plants tend to have evolved a relatively larger gene family; e.g., the $N F Y$ gene family has 10 members in Arabidopsis ${ }^{52}, 8$ in rice $^{53}, 10$ in wheat $^{54}$, and 10 in tomato ${ }^{55}$. Gene replication may contribute to large numbers of gene families that allow functional compensation or buffering against individual mutations, subsequently leading to functional redundancy ${ }^{56}$. The F-box protein family also has a large amount of members, with almost 700 predicted genes in Arabidopsis. However, single mutations or combinations of mutations in pollen-specific F-box genes did not lead to gametophytic lethality, suggesting functional redundancy among the F-box protein-coding genes ${ }^{57}$. In plant viruses such as tobacco etch potyvirus, genotypes with genetic redundancy suffered less from the effects of deleterious mutations and showed relatively minor changes in pathogenicity $^{58}$. Accordingly, the expression of alternative members of the NFYA gene family, e.g., SINFYA1.1, SINFYA3.1, and SINFYA8, was significantly higher in the SINFYA10 RNAi lines than in the wild type (Supplementary Fig. S8). Thus, we hypothesized that the relative stability of plant performance in the SINFYA10 RNAi transgenic lines might be in part due to functional redundancy of NFYA gene family members in tomato.

\section{Conclusion}

We used the SlGME1 promoter to isolate a CCAAT-box transcription factor, SINFYA10, which could modulate AsA biosynthesis at multiple sites. SINFYA10 exerted a negative 
effect on SlGME1 and SlGGP1 expression and AsA accumulation. SINFYA10-overexpressing lines showed a lower antioxidant capacity, with lower AsA content. The identification of potential interacting proteins involved in the heterotrimer complex will provide deeper insight into SINFYA1O regulation of AsA biosynthesis.

\section{Acknowledgements}

This work was supported by grants from the National Key Research \& Development Plan (2018YFD1000800, 2016YFD0100204-21); National Natural Science Foundation of China (31991182, 31972426, and 31672166);

Fundamental Research Funds for the Central Universities (2662018PY073) and Wuhan Frontier Projects for Applied Foundation (2019020701011492)

\section{Conflict of interest}

The authors declare that they have no conflict of interest.

Supplementary Information accompanies this paper at (https://doi.org/ 10.1038/s41438-020-00418-6).

Received: 18 March 2020 Revised: 15 August 2020 Accepted: 18 August 2020

Published online: 01 December 2020

\section{References}

1. Macknight, R. C. et al. Increasing ascorbate levels in crops to enhance human nutrition and plant abiotic stress tolerance. Curr. Opin. Biotechnol. 44, 153-160 (2017).

2. Drisko, J. A. et al. Treatment of pancreatic cancer with intravenous vitamin C: a case report. Anticancer Drugs 29, 373-379 (2018).

3. Kocot, J. et al. Does Vitamin C influence neurodegenerative diseases and psychiatric disorders? Nutrients 9, 659 (2017).

4. Wheeler, G. L. et al. The biosynthetic pathway of vitamin C in higher plants. Nature 393, 365-369 (1998).

5. Davey, M. W. et al. Ascorbate biosynthesis in Arabidopsis cell suspension culture. Plant Physiol. 121, 535-543 (1999).

6. Wolucka, B. A. et al. GDP-mannose 3',5'-epimerase forms GDP-L-gulose, a putative intermediate for the de novo biosynthesis of vitamin $C$ in plants. J. Biol. Chem. 278, 47483-47490 (2003).

7. Lorence, A. et al. myo-inositol oxygenase offers a possible entry point into plant ascorbate biosynthesis. Plant Physiol. 134, 1200-1205 (2004).

8. Conklin, P. L. et al. Genetic evidence for the role of GDP-mannose in plant ascorbic acid (vitamin C) biosynthesis. Proc. Natl Acad. Sci. USA 96, 4198-4203 (1999).

9. Wolucka, B. A. et al. Partial purification and identification of GDP-mannose 3",5"-epimerase of Arabidopsis thaliana, a key enzyme of the plant vitamin C pathway. Proc. Natl Acad. Sci. USA 98, 14843-14848 (2001).

10. Dowdle, J. et al. Two genes in Arabidopsis thaliana encoding GDP-L-galactose phosphorylase are required for ascorbate biosynthesis and seedling viability. Plant J. 52, 673-689 (2007).

11. Laing, W. A. et al. A highly specific L-galactose-1-phosphate phosphatase on the path to ascorbate biosynthesis. Proc. Natl Acad. Sci. USA 101, 16976-16981 (2004).

12. Gatzek, S. et al. Antisense suppression of L-galactose dehydrogenase in Arabidopsis thaliana provides evidence for its role in ascorbate synthesis and reveals light modulated I-galactose synthesis. Plant J. 30, 541-553 (2002).

13. Imai, $T$. et al. L-galactono- $\gamma$-lactone dehydrogenase from sweet potato: purification and CDNA sequence analysis. Plant Cell Physiol. 39, 1350-1358 (1998).

14. Watanabe, K. et al. Characterization of a GDP-D-mannose 3',5'-epimerase from rice. Phytochemistry 67, 338-346 (2006).

15. Stevens, R. et al. Candidate genes and quantitative trait loci affecting fruit ascorbic acid content in three tomato populations. Plant Physiol. 143, 1943-1953 (2007).

16. Zhang, C. et al. Overexpression of SIGMEs leads to ascorbate accumulation with enhanced oxidative stress, cold, and salt tolerance in tomato. Plant Cell Rep. 30, 389-398 (2011).
17. Gilbert, L. et al. GDP-D-mannose 3,5-epimerase (GME) plays a key role at the intersection of ascorbate and non-cellulosic cell-wall biosynthesis in tomato. Plant J. 60, 499-508 (2009).

18. Mounet-Gilbert, L. et al. Two tomato GDP-D-mannose epimerase isoforms involved in ascorbate biosynthesis play specific roles in cell wall biosynthesis and development. J. Exp. Bot. 67, 4767-4777 (2016).

19. Ntagkas, N. et al. Light regulates ascorbate in plants: an integrated view on physiology and biochemistry. Environ. Exp. Bot. 147, 271-280 (2018).

20. Yabuta, Y. et al. Light regulation of ascorbate biosynthesis is dependent on the photosynthetic electron transport chain but independent of sugars in Arabidopsis. J. Exp. Bot. 58, 2661-2671 (2007).

21. Li, H. et al. Differentially expressed protein and gene analysis revealed the effects of temperature on changes in ascorbic acid metabolism in harvested tea leaves. Hortic. Res. 5, 65 (2018).

22. Jin, P. F. et al. Rapid determination of thiamine, riboflavin, niacinamide, pantothenic acid, pyridoxine, folic acid and ascorbic acid in vitamins with minerals tablets by high-performance liquid chromatography with diode array detector. J. Pharm. Biomed. Anal. 70, 151-157 (2012).

23. Tsaniklidis, G. et al. Induced parthenocarpic cherry tomato fruits did not shown significant differences in l-ascorbate content but showed different pattern in GallDH and GME expression. Plant Growth Regul. 68, 493-502 (2012).

24. Wolucka, B. A. et al. Methyl jasmonate stimulates the de novo biosynthesis of vitamin C in plant cell suspensions. J. Exp. Bot. 56, 2527-2538 (2005).

25. Mir, M. A. et al. Jasmonic acid ameliorates alkaline stress by improving growth performance, ascorbate glutathione cycle and glyoxylase system in maize seedlings. Sci. Rep. 8, 2831 (2018).

26. Kerchev, P. I. et al. The transcription factor ABI4 Is required for the ascorbic acid-dependent regulation of growth and regulation of jasmonatedependent defense signaling pathways in Arabidopsis. Plant Cell 23, 3319-3334 (2011).

27. Zhang, W. et al. AMR1, an Arabidopsis gene that coordinately and negatively regulates the mannose/l-galactose ascorbic acid biosynthetic pathway. Plant Physiol. 150, 942-950 (2009).

28. Wang, J. et al. Arabidopsis CSN5B interacts with VTC1 and modulates ascorbic acid synthesis. Plant Cell 25, 625-636 (2013).

29. Zhang, Z. et al. The ethylene response factor AtERF98 enhances tolerance to salt through the transcriptional activation of ascorbic acid synthesis in Arabidopsis. Plant J. 71, 273-287 (2012).

30. Hu, T. et al. The tomato HD-Zip I transcription factor SIHZ24 modulates ascorbate accumulation through positive regulation of the D-mannose/Lgalactose pathway. Plant J. 85, 16-29 (2016).

31. Sawake, S. et al. KONJAC1 and 2 are key factors for GDP-mannose generation and affect L-ascorbic acid and glucomannan biosynthesis in Arabidopsis. Plant Cell 27, 3397-3409 (2015).

32. $\mathrm{Li}, \mathrm{Y}$. et al. The $\mathrm{C} 2 \mathrm{H} 2$ zinc-finger protein SIZF3 regulates AsA synthesis and salt tolerance by interacting with CSN5B. Plant Biotechnol. J. 16, 1201-1213 (2017).

33. Laing, W. A. et al. An upstream open reading frame is essential for feedback regulation of ascorbate biosynthesis in Arabidopsis. Plant Cell 27, 772-786 (2015).

34. Laloum, T. et al. CCAAT-box binding transcription factors in plants: $Y$ so many? Trends Plant Sci. 18, 157-166 (2013).

35. Cagliari, A. et al. New insights on the evolution of Leafy cotyledon1 (LEC1) type genes in vascular plants. Genomics 103, 380-387 (2014).

36. Dolfini, D. et al. NF-Y and the transcriptional activation of CCAAT promoters. Crit. Rev. Biochem. Mol. Biol. 47, 29-49 (2012).

37. Qu, B. et al. A wheat CCAAT box-binding transcription factor increases the grain yield of wheat with less fertilizer input. Plant Physiol. 167, 411-423 (2015).

38. $\mathrm{Bi}$, C. et al. Overexpression of the transcription factor NF-YC9 confers abscisic acid hypersensitivity in Arabidopsis. Plant Mol. Biol. 95, 425-439 (2017).

39. Li, W. X. et al. The Arabidopsis NFYA5 transcription factor is regulated transcriptionally and posttranscriptionally to promote drought resistance. Plant Cell 20, 2238-2251 (2008).

40. Ma, X. et al. Overexpression of wheat NF-YA10 gene regulates the salinity stress response in Arabidopsis thaliana. Plant Physiol. Biochem. 86, 34-43 (2015).

41. Siriwardana, C. L. et al. NUCLEAR FACTOR Y, Subunit A (NF-YA) proteins positively regulate flowering and act through FLOWERING LOCUS T. PLOS Genet. 12, e1006496 (2016).

42. Kim, S. K. et al. OsNF-YC2 and OsNF-YC4 proteins inhibit flowering under longday conditions in rice. Planta 243, 563-576 (2016). 
43. Cao, S. et al. A distal CCAAT/NUCLEAR FACTOR Y complex promotes chromatin looping at the FLOWERING LOCUS T promoter and regulates the timing of flowering in Arabidopsis. Plant Cell 26, 1009-1017 (2014).

44. Hellens, R. P. et al. Transient expression vectors for functional genomics, quantification of promoter activity and RNA silencing in plants. Plant Methods 1, 13 (2005).

45. Wang, Y. et al. Soybean miR172c targets the repressive AP2 transcription factor NNC1 to activate ENOD40 expression and regulate nodule initiation. Plant Cell 26, 4782-4801 (2014).

46. Dobisova, T. et al. Light controls cytokinin signaling via transcriptional regulation of constitutively active sensor histidine kinase CKI1. Plant Physiol. 174, 387-404 (2017).

47. Stevens, R. et al. Technique for rapid, small-scale analysis of vitamin C levels in fruit and application to a tomato mutant collection. J. Agric. Food Chem. 54, 6159-6165 (2006).

48. Yoo, S. D. et al. Arabidopsis mesophyll protoplasts: a versatile cell system for transient gene expression analysis. Nat. Protoc. 2, 1565-1572 (2007).

49. Lescot, M. et al. PlantCARE, a database of plant cis-acting regulatory elements and a portal to tools for in silico analysis of promoter sequences. Nucleic Acids Res. 30, 325-327 (2002).

50. Tao, Q. et al. The TIE1 transcriptional repressor links TCP transcription factors with TOPLESS/TOPLESS-RELATED corepressors and modulates leaf development in Arabidopsis. Plant Cell 25, 421-437 (2013).
51. Garipov, A. et al. NF-YA underlies EZH2 upregulation and is essential for proliferation of human epithelial ovarian cancer cells. Mol. Cancer Res. 11, 360-369 (2013).

52. Gusmaroli, G. et al. Regulation of novel members of the Arabidopsis thaliana CCAAT-binding nuclear factor $Y$ subunits. Gene 283, 41-48 (2002).

53. Thirumurugan, $T$. et al. Identification, characterization and interaction of HAP family genes in rice. Mol. Genet. Genomics 279, 279-289 (2008).

54. Stephenson, T. J. et al. Genome-wide identification and expression analysis of the NF-Y family of transcription factors in Triticum aestivum. Plant Mol. Biol. 65 77-92 (2007).

55. Li, S. et al. Genome-wide analysis of tomato NF-Y factors and their role in fruit ripening. BMC Genomics 17, 36 (2016).

56. Chapman, B. A. et al. Buffering of crucial functions by paleologous duplicated genes may contribute cyclicality to angiosperm genome duplication. Proc. Natl Acad. Sci. 103, 2730-2735 (2006).

57. Ikram, S. et al. Functional redundancy and/or ongoing pseudogenization among F-box protein genes expressed in Arabidopsis male gametophyte. Plant Reprod. 27, 95-107 (2014).

58. Ambros, S. et al. Engineered functional redundancy relaxes selective constraints upon endogenous genes in viral RNA genomes. Genome Biol. Evolution 10, 1823-1836 (2018). 